\title{
Electret formation in transition metal oxides by electrochemical amorphization
}

Yong-Jin Kim (iD) ${ }^{1,2}$ and Chan-Ho Yang ${ }^{1,2,3}$

\begin{abstract}
Transition metal oxides (TMOs) are an important class of materials that show a wide range of functionalities involving spin, charge, and lattice degrees of freedom. The strong correlation between electrons in $d$-orbitals and the multivalence nature give rise to a variety of exotic electronic states ranging from insulator to superconductor and cause intriguing phase competition phenomena. Despite a burst of research on the multifarious functionalities in TMOs, little attention has been paid to the formation and integration of an electret-a type of quasi-permanent electric field generator useful for nanoscale functional devices as an electric counterpart to permanent magnets. Here, we find that an electret can be created in $\mathrm{LaMnO}_{3}$ thin films by tip-induced electric fields, with a considerable surface height change, via solid-state electrochemical amorphization. The surface charge density of the formed electret area reaches $\sim 400 \mathrm{nC} \mathrm{cm}^{-2}$ and persists without significant charge reduction for more than a year. The temporal evolution of the surface height, charge density, and electric potential are systematically examined by scanning probe microscopy. The underlying mechanism is theoretically analyzed based on a drift-diffusion-reaction model, suggesting that positively charged particles, which are likely protons produced by the dissociation of water, play crucial roles as trapped charges and a catalysis to trigger amorphization. Our finding opens a new horizon for multifunctional TMOs.
\end{abstract}

\section{Introduction}

Electrets, materials that retain quasi-permanent electric charges or dipole moments generating internal and external electric fields, have been intensively studied because of their wide range of applications, such as in microphones, xerography devices, sensors, actuators, energy harvesting generators ${ }^{1,2}$, and silicon photovoltaic devices $^{3-5}$. Many studies have been conducted on polymer-based organic electrets, such as polytetrafluoroethylene (PTFE) and cyclic transparent optical polymer (CYTOP), due to their soft nature ${ }^{2,6,7}$ and silicon-based inorganic materials, such as $\mathrm{SiO}_{2}, \mathrm{Si}_{3} \mathrm{~N}_{4}$ and their composite layers, because of their compatibility with the silicon semiconductor industry ${ }^{1-5}$. In addition, various

\footnotetext{
Correspondence: Chan-Ho Yang (chyang@kaist.ac.kr)

'Department of Physics, Korea Advanced Institute of Science and Technology (KAIST), Daejeon 34141, Republic of Korea

${ }^{2}$ Center for Lattice Defectronics, KAIST, Daejeon 34141, Republic of Korea

Full list of author information is available at the end of the article.
}

binary oxide electrets have been introduced for passivating silicon surfaces and solar cell applications ${ }^{3-5,8-10}$.

Meanwhile, complex transition metal oxides (TMOs) have spurred tremendous research interest due to their various functionalities, such as colossal magnetoresistance, high-temperature superconductivity, and electrochemical catalytic activity ${ }^{11-13}$. However, the implementation of an electret in complex TMOs has hitherto been little explored, even though an electret hybridized with the existing functionalities in TMOs enables us to envision new functional architectures and properties. It must be explored whether TMOs $\left(\mathrm{LaMnO}_{3}\right.$ (LMO) in our case) can be a base material to generate electrets. The possibility of multiple oxidation (valence) states of transition metal ions offers rich phase diagrams and competing physical properties susceptibly tuned by external perturbations ${ }^{11,12,14,15}$ and facilitates chemical complex ion formation ${ }^{16-18}$ and catalytic activity ${ }^{13}$. However, the feature of variable oxidation states is also a reason hindering the formation of electrets in TMOs due to fast 
neutralization through electronic screening and consequent valence state changes.

Our strategy to tackle the problem is to increase the disorder of the system, in which the electronic states are expected to be strongly localized ${ }^{19}$, and therefore, electronic states containing excess charges can be sustained for a long time. We used electrochemical amorphization realized through scanning probe lithography $(\mathrm{SPL})^{20}$, whereby the electrochemical reaction can be activated in a tip-sample geometry involving the natural formation of a water bridge between the biased tip and the sample surface through capillary condensation, forming an electrochemical cell ${ }^{21,22}$. Although SPL studies on TMOs have focused on electronic conduction ${ }^{23-25}$ and topographic properties $^{21,26}$, studies on emergent electrostatic states in complex TMOs have been little explored. Since an electret must have a high density of electric charges that is preserved for an extended period of time, comprehensive investigations of the electrostatic charges as well as their retention are demanded to explore this idea.

As a target material, we employed LMO, which is an insulator with (лт0)-type ordering of anisotropic $3 d e_{g}$ orbitals in $\mathrm{Mn}^{3+}\left(t_{2 g}^{3} e_{g}^{1}\right)$ accompanied by the cooperative Jahn-Teller distortion ${ }^{27,28}$. Appropriate hole doping of the compound modulates the electronic and magnetic natures, including the colossal magnetoresistance phenomenon and phase separation ${ }^{11,15,29-34}$. From the perspective of chemistry, LMO is receiving attention due to its high electrocatalytic activity, which originates from single electron occupancy in Mn $e_{g}$ orbitals and high covalence between the transition metal ion and oxygen anions ${ }^{13}$. Such electrochemically active materials are good candidates for amorphization via $\mathrm{SPL}^{35-37}$.

In this work, we report an experimental demonstration of an electret in TMOs. Highly stabilized charge patterns are successfully realized in a LMO thin film using electrochemical amorphization triggered by tip-induced electric fields. We confirm the existence of electric fields outside the film and quantify the electrostatic surface potential by scanning probe microscopy techniques. The surface charge density is estimated to be on a similar order of magnitude to those of commercially available electrets, and the field associated with the charge is verified to persist for more than a year. A simulation study of our drift-diffusion-reaction model in comparison with various detailed experimental features, such as the natural height expansion, suggests that externally injected protons during the electroforming process act as an electrochemical catalyst to convert the crystalline LMO to an amorphous phase in the diffusion-limited regime while the concentration of the mobile catalytic species diminishes with time. The combined theoretical and experimental studies provide an in-depth understanding of the electrochemical reaction in LMO.

\section{Materials and methods}

\section{Synthesis of LMO thin films}

LMO thin films with a $\mathrm{La}_{0.7} \mathrm{Sr}_{0.3} \mathrm{MnO}_{3}$ bottom electrode were deposited on a $(110)_{O}$-oriented DSO substrate (CrysTec $\mathrm{GmbH}$ ) using pulsed laser deposition with a $\mathrm{KrF}$ excimer laser $(\lambda=248 \mathrm{~nm})$. Growth was performed at a temperature of $950^{\circ} \mathrm{C}$ and an oxygen partial pressure of $10 \mathrm{mTorr}$. The laser fluence was $0.24 \mathrm{~J} \mathrm{~cm}^{-2}$ with a repetition rate of $10 \mathrm{~Hz}$. After deposition, the films were cooled to room temperature at a rate of $10^{\circ} \mathrm{C} \mathrm{min}{ }^{-1}$ in a vacuum environment $\left(\sim 10^{-6}\right.$ Torr) to resolve the cation vacancy issue by reducing excess oxygen. Details of the orbital ordering in the films can be found elsewhere ${ }^{28}$.

\section{Characterization of surface morphology and surface charge}

The surface topographies of the pristine LMO thin film were characterized using atomic force microscopy (AFM) (Veeco-DI Multimode V equipped with a Nanoscope controller V) with conducting Pt-coated Si tips (HQ: NSC35/Pt, MikroMasch). The phase shift maps were obtained using electrostatic force microscopy (EFM) in interleave mode after measuring the surface topographies in tapping mode. In the EFM measurements, the tip floated $50 \mathrm{~nm}$ above the sample surface. Kelvin probe force microscopy (KPFM) measurements were carried out to determine the surface potentials of the sample over time, and the surface morphologies were simultaneously obtained. The tip floated $50 \mathrm{~nm}$ above the sample surface in KPFM measurements. All measurements were performed at a tip sweeping speed of $5 \mu \mathrm{m} \mathrm{s}^{-1}$.

\section{Transmission electron microscopy (TEM) measurement}

To characterize the cross-sectional crystallographic structure and perform EELS measurements, we employed an aberration-corrected Titan cubed G2 60-300 (FEI) equipped with double $\mathrm{Cs}$ correctors and a monochromator. The samples were prepared by the focused ion beam (FIB) method using a FEI Quanta 3D FEG. Before the FIB process, Ti and Pt were deposited by DC magnetron sputtering to protect the film from the Ga ion beam during the focused ion beam (FIB) lift-out process.

\section{Drift-diffusion-reaction model}

To reflect the volume expansion due to the chemical reaction in our model, which will be explained later using Eqs. (2)-(5), we considered the increment of the spatial element $(\Delta z)$ after time $\Delta t$ at position $z$ as follows:

$$
\Delta z=\frac{\lambda-1}{\rho_{\mathrm{LMO}}} k \mathrm{n}_{\mathrm{P}} \mathrm{n}_{\mathrm{LMO}} \Delta t
$$

where $\rho_{\mathrm{LMO}}$ represents the number density, $\sim 1.6 \times 10^{22}$ $\mathrm{cm}^{-3}$, of the crystalline LMO unit cells. The space-chargeinduced electric field was calculated on the assumption that 
the infinite sheet charge density varies along the $z$-axis according to the Gauss law, i.e., $E(z, t)=$ $\int_{0}^{z} d z^{\prime} e\left(\mathrm{n}_{\mathrm{P}}\left(z^{\prime}, t\right)+\gamma \mathrm{n}_{\mathrm{a}-\mathrm{PLMO}}\left(z^{\prime}, t\right)\right) / 2 \varepsilon-\int_{z}^{h} d z^{\prime} e\left(\mathrm{n}_{\mathrm{P}}\left(z^{\prime}, t\right)+\right.$ $\left.\gamma \mathrm{n}_{\mathrm{a}-\mathrm{PLMO}}\left(z^{\prime}, t\right)\right) / 2 \varepsilon$, where $\varepsilon$ is the dielectric constant $10 \varepsilon_{0}$ and $h$ is the film thickness. The electric potential was calculated to be $V(z, t)-V(0, t)=-\int_{0}^{z} E\left(z^{\prime}, t\right) d z^{\prime}$. We categorized the overall simulation processes as two steps before and after the tip bias was turned off at $t=0$. In the preprocess step $(t<0)$, we started with a surface charge density of positively charged particles of $\sim 3 \times 10^{12} \mathrm{e} \mathrm{cm}^{-2}$ with a uniform depth of $8 \mathrm{~nm}$, we assumed that the chemical reaction coefficient was 10,000 times enhanced as a result of the external field of the biased tip, and we relaxed the charge distribution until the chemical reaction was completed for $\sim 5 \mathrm{~ms}$, which was short enough to neglect the diffusion effect. In the step at $t>0$ without external fields, we tracked the chemical process and diffusion to monitor the natural expansion and surface potential change using the charge configuration saturated in the preprocess step.

\section{Results and discussion}

\section{Electret formation}

We prepared an $\sim 20$-nm-thick LMO thin film with an 2-nm-thick $\mathrm{La}_{0.7} \mathrm{Sr}_{0.3} \mathrm{MnO}_{3}$ bottom electrode on a $(110)_{O}$-oriented $\mathrm{DyScO}_{3}$ (DSO) substrate (where the subscript $O$ stands for the orthorhombic index) by pulsed laser deposition under a high-temperature growth condition that maintains the orbital ordering even in thin films ${ }^{28}$. The electric voltages and potentials mentioned in this paper are defined as relative values with respect to the bottom electrode. Figure 1a shows a topographic image of a pristine region (PR) of the film measured by AFM. An atomically flat surface with stripe twin domains along $[1 \overline{1} 0]_{O}$ was detected because of the anisotropic misfit strain in the LMO film grown on the orthorhombic DSO substrate $^{38}$. The crystal structure was confirmed by a conventional X-ray $\theta-2 \theta$ scan (Supplementary Fig. 1). No noticeable impurity peaks in addition to the film and substrate peaks were detected in the wide range of scan angles.

To form the charge patterns, we used conductive Ptcoated Si tips. The typical radius of a tip was $\sim 20 \mathrm{~nm}$, and the distance between the cantilever and the tip apex was $12-18 \mu \mathrm{m}$. A $5 \times 5 \mu \mathrm{m}^{2}$ region was scanned by a conductive tip biased at a forming voltage $\left(V_{\mathrm{Ef}}\right)$ of $+10 \mathrm{~V}$ (the electric field strength was estimated to be $\sim 5 \times 10^{6} \mathrm{~V} \mathrm{~cm}^{-1}$ considering the film thickness of $20 \mathrm{~nm}$ ) while holding direct contact between the tip and sample surface under ambient conditions. In a scan, the trajectory of the tip draws a zigzag path in which the tip moves forward and backward along the fast scan direction at a constant speed of $5 \mu \mathrm{m} \mathrm{s}^{-1}$, while the tip moves downward along the slow scan direction at a rate of 1 line $\mathrm{s}^{-1}$. The region was formed by scanning $2 \times 128$ lines.
The surface topography and electrostatic state in the electrically formed region (EfR) were characterized by AFM, EFM and KPFM, as shown in Fig. 1b-d. Figure 1b, c shows the simultaneously obtained surface topography and EFM phase map relative to the PR under $V_{\mathrm{EFM}}=+$ $4 \mathrm{~V}$ (here, " $V_{\mathrm{EFM}}$ " represents the voltage applied to the tip during the EFM measurement). The EfR on a square and protruding region had a different electrostatic state from the PR. In terms of the topography, the EfR was $\sim 8 \mathrm{~nm}$ higher than the PR, as shown in the line profile in Fig. 1e. The sharp boundary, flat plateau, and nearly preserved surface structure validate that a distinctive and uniform forming process was performed. The enhanced spiky surface topography in the EfR is attributed to crystal orientation-dependent chemical activity ${ }^{39}$. To elucidate whether the SPL was activated through a water bridge between the tip and the sample surface, we investigated the humidity-dependent height expansion (Supplementary Fig. 2) and found that a higher height was formed at higher humidity. Water molecules can be decomposed in electric fields, producing protons. The humidity dependence suggests that the height change most likely involves the penetration of the protons created by water splitting into the underneath material ${ }^{21}$. The positive tip voltage generates a downward electric field that causes the positive ions to migrate toward the material. In contrast, when the opposite polarity electric field was used, no volume expansion was detected (Supplementary Fig. 3). This proton injection resembles the potassium ion injection into silicon oxides for electret formation ${ }^{40,41}$ and the field effect surface passivation of solar cells ${ }^{42}$.

To confirm the electrostatic states of the film, we performed EFM with various bias voltages, i.e., $V_{\mathrm{EFM}}{ }^{43-45}$. As shown in Fig. 1f, g, we confirmed that the phase shift in the EfR clearly shows a linear dependence on $V_{\mathrm{EFM}}$. The linear dependence of the cantilever phase shift on $V_{\mathrm{EFM}}$ reflects an interaction between the space charges on the sample surface and the cantilever tip. Since a positive (negative) phase shift reveals a repulsive (attractive) interaction $^{45}$, we definitely concluded that excessive positive charges exist in the formed area. Meanwhile, a purely quadratic dependence was observed in the PR (Supplementary Fig. 4), which is due to a capacitive interaction between the neutral sample and the tip.

To determine the surface charge density in the EfR, we measured the electric potential at the top surface of the film relative to the bottom electrode using $\mathrm{KPFM}^{46}$, as shown in Fig. 1d, h. Since the radius of the tip $(\sim 20 \mathrm{~nm})$ was much smaller than the EfR, we were able to estimate the surface charge density $(\sigma)$ using the simple infinitearea charge density model. The value of $\sigma$ was estimated to be $\sigma=2 \varepsilon V / h^{\prime} \sim 400 \mathrm{nC} \mathrm{cm}^{-2}$, where $V$ represents the measured surface potential, $\varepsilon$ is the dielectric constant of $\mathrm{LMO}^{47}$, and $h^{\prime}$ is the effective film thickness, which is 

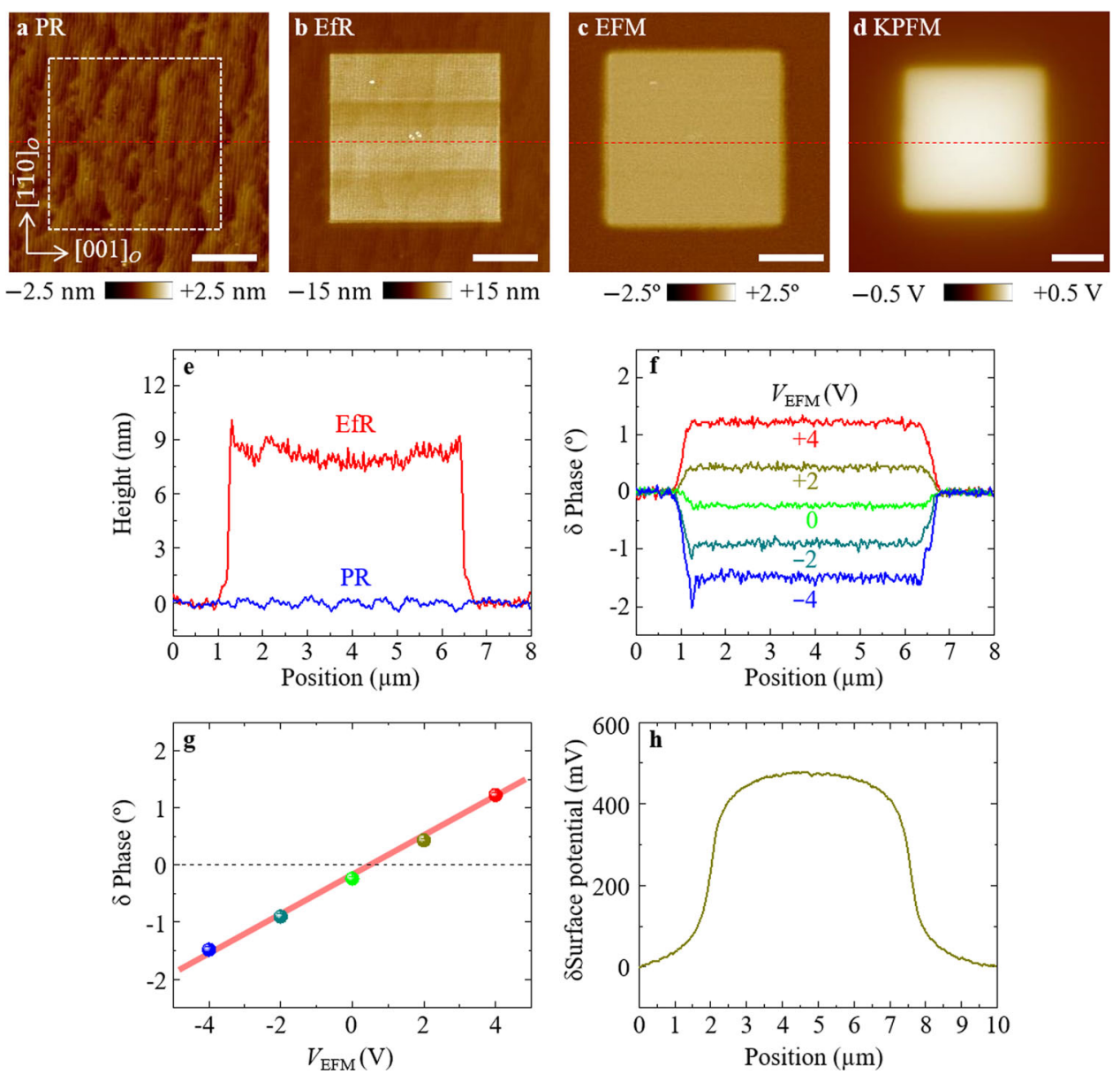

Fig. 1 Formation of the charge pattern. a Surface topographic image of the pristine film. The white boxed area indicates the region scanned by a positively biased tip. b Topographic image after the electroforming process. c Relative EFM phase map measured under $V_{\text {EFM }}=+4 \mathrm{~V}$. $\mathbf{d}$ Relative surface potential map in the same region. e Height profiles before (blue) and after (red) the electroforming process. $\mathbf{f}$ Relative EFM phase profiles and $\mathbf{g}$ average relative phase values in the electroformed regions for various $V_{\text {EFM }}$ values. The light red line is a guideline for the eye. $\mathbf{h}$ Profile data of the relative surface potential. All scale bars represent $2 \mu \mathrm{m}$, and all profile data were obtained from the corresponding red dotted lines in a-d.

estimated to be $\sim 20 \mathrm{~nm}$ smaller than the total film thickness of the electroformed area $(\sim 28 \mathrm{~nm})$. This is because not all charges reside on the top surface with an infinitesimal width but are distributed within the electroformed film with a charge depth profile that will be addressed later in this paper. The estimated value of $\sigma$ corresponds to $\sim 3 \times 10^{12} e \mathrm{~cm}^{-2}$ ( $e$ is the elementary charge), which is on the same order of magnitude as the representative high charge density reported in CYTOP and $\mathrm{SiO}_{2}$ electrets ${ }^{2}$.

To investigate the forming voltage $V_{\mathrm{Ef}}$ dependence, we fabricated a stepwise pattern using various $V_{\mathrm{Ef}}$ from $+1 \mathrm{~V}$ to $+10 \mathrm{~V}$ and measured the topographic and electrostatic states immediately after formation. Figure 2 shows the surface topographies and corresponding relative EFM phase maps measured at $V_{\mathrm{EFM}}=4 \mathrm{~V}$. Clearly, the higher the $V_{\mathrm{Ef}}$ applied, the higher the heights fabricated. The changes in height for $V_{\mathrm{Ef}}<3 \mathrm{~V}$ were not clearly detected, which is ascribed to the weak activity of the water decomposition. Meanwhile, the EFM phase becomes saturated at $V_{\mathrm{Ef}}>4 \mathrm{~V}$ (Fig. 2c, g and Supplementary Fig. 5). Since the EFM phases reflect the amount of injected charges, the saturation behavior reveals that the number of charges is limited by the finite amount of water condensed between the tip and the sample. In contrast, the height of the EfR still shows a quadratic increase for $V_{\mathrm{Ef}}>$ $4 \mathrm{~V}$ (the inset of Fig. 2f) because the chemical reaction becomes more active under higher external electric fields.

To determine the long-term stability of the EfR as a requirement of electrets, we measured the height and the EFM phase again on the 449th day after formation. Comparing the heights on the 0th and 449th days, an additional height increase was detected in the EfR for the $V_{\mathrm{Ef}}>4 \mathrm{~V}$ region (Fig. 2b, f). The sample had been stored in ambient conditions. Remaining charges were still detected in the EfRs formed with $V_{\mathrm{Ef}}>4 \mathrm{~V}$, while the charges were 

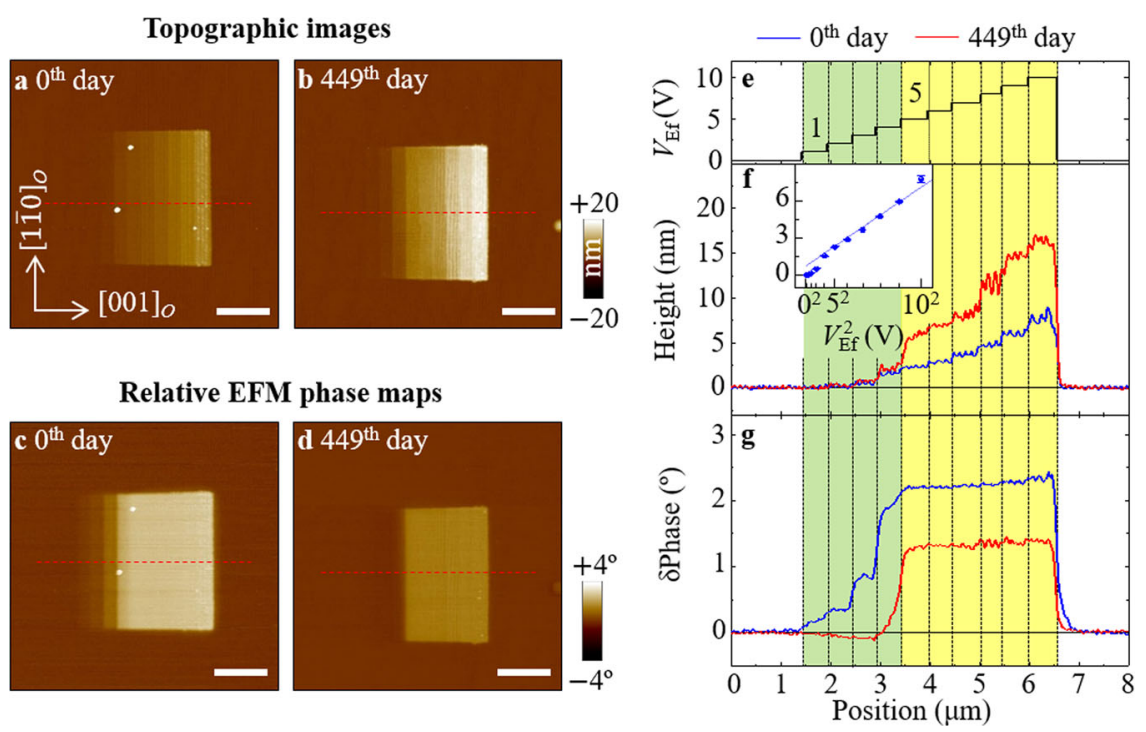

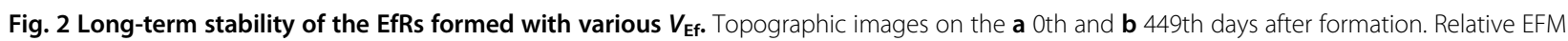
phase maps on the $\mathbf{c}$ 0th and $\mathbf{d}$ 449th days after formation, measured under $V_{\mathrm{EFM}}=4 \mathrm{~V}$. e Applied voltages $\left(V_{\mathrm{EF}}\right)$ in the forming process, and corresponding $\mathbf{f}$ height profiles and $\mathbf{g}$ relative EFM phase profiles on the 0th and 449th days. The inset of $\mathbf{f}$ represents the average heights on the 0th day for a given $V_{\mathrm{Ef}}$, with a light blue guideline for the eye. The greenish (yellowish) region indicates $V_{\mathrm{Ef}}=1 \mathrm{~V}$ to $4 \mathrm{~V}\left(V_{\mathrm{Ef}}=5 \mathrm{~V}\right.$ to $\left.10 \mathrm{~V}\right)$ regions. The profile data were obtained along the horizontal red dotted lines on the corresponding images. All scale bars in a-d represent $2 \mu \mathrm{m}$.

nearly neutralized for the $V_{\mathrm{Ef}}<4 \mathrm{~V}$ regions (Fig. $2 \mathrm{~d}$, g and Supplementary Fig. 5). The behaviors of the height and residual charges indicate that in the region where an additional height increase occurred, the injected charges are retained for many days, and in the other regions, the charges easily vanish. Therefore, long-term stability of the charge patterns was achieved with $V_{\mathrm{Ef}}>4 \mathrm{~V}$, and the remaining charges activated the additional height expansion. We presume that the additional height increase occurs via long-term diffusion of the injected protons and their chemical reaction with the LMO. The additional expansion provides useful insights into the detailed features of the chemical reaction, which will be discussed below.

\section{Crystallographic structures}

To confirm the crystallographic structure beneath the surface after the formation, we obtained high-angle annular dark-field (HAADF) images including a PR and an EfR. Figure 3a shows low magnification HAADF images containing both regions. The height difference between the PR and EfR was clearly recognized, and a color contrast between both regions near the surface was observed. Figure $3 \mathrm{~b}$ and $\mathrm{c}$ presents atomic images near the film surfaces of the PR and EfR, respectively. The PR had regular atomic arrangements, while the EfR had irregular atomic arrangements, indicating the formation of an amorphous phase. Figure 3d, e depicts fast Fourier transform (FFT) images of the atomic arrangements within the white dotted boxes in Fig. 3b, c. The FFT image of the EfR indicated an amorphous phase, while clear periodic diffraction peaks were observed in the PR. Electrochemical amorphization has often been observed in TMOs due to redox reactions under high electric currents or potentials ${ }^{35-37}$. Water-mediated amorphization via hydrogen injection was also reported during $\mathrm{SPL}^{21}$. Although the mechanism of amorphization is not yet clearly understood, we hypothesize that the stabilization of the electret properties can be ascribed to the increased disorder in the chemical arrangement and injection of extrinsic impurities during the electroforming process. The electronic states of the amorphous phase are highly expected to be spatially localized as a result of the Anderson localization mechanism ${ }^{19}$, and the injected impurities, such as protons, would induce deep trap levels $^{48}$, which hinder charge neutralization. Local electrical measurements (Supplementary Fig. 6) evidently show that the EfR had a higher resistance than the PR due to the amorphization. We also note that amorphization caused a volume expansion of $\sim 200 \%$, thereby producing relatively dark contrast in the Z-contrast images. Such low ionic density enables us to speculate a porous structure at the atomic level, although we can hardly observe the details due to random averaging of the atomic arrangements through the foil thickness of the TEM specimen along the zone axis.

\section{Drift-diffusion-reaction model}

To gain deeper insight into the underlying mechanism, we theoretically constructed a drift-diffusion-reaction 


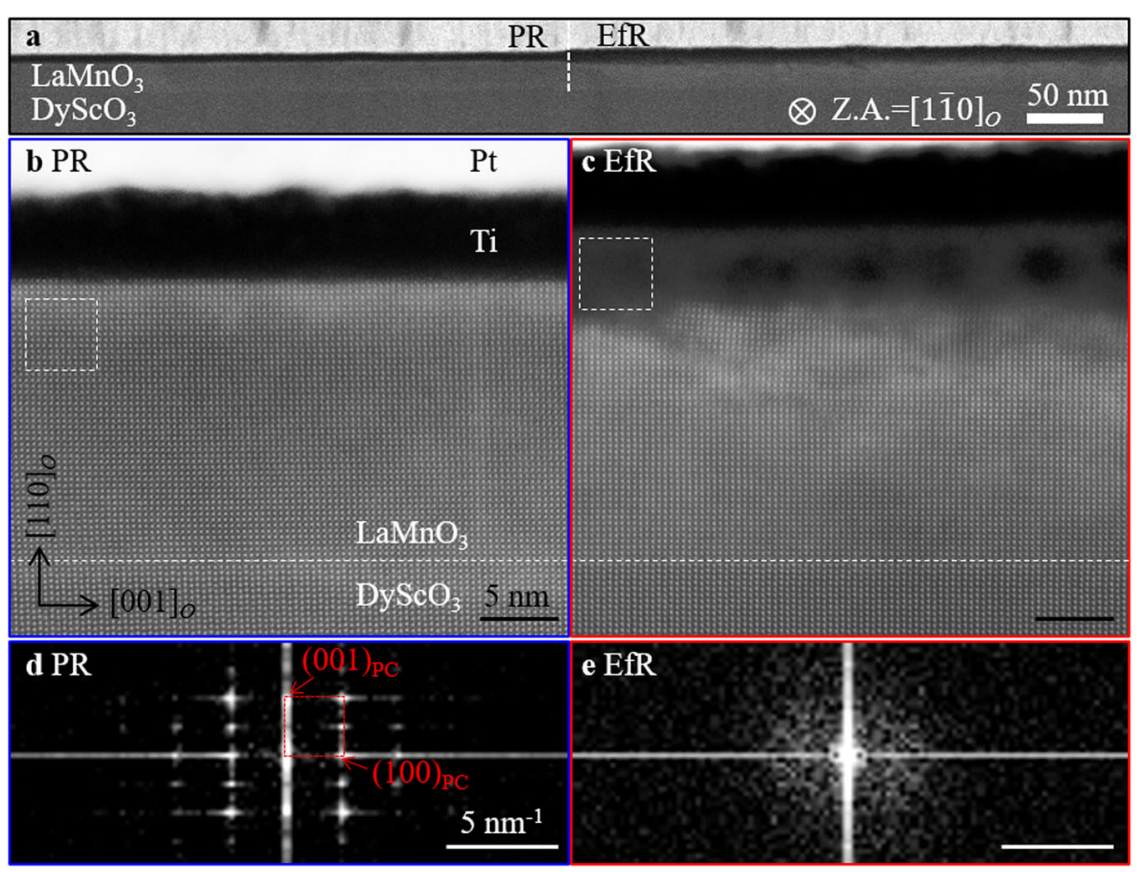

Fig. 3 HAADF images of a cross-sectional LMO thin film containing the boundary of an EfR and its neighboring PR. a Large area image of the film and substrate, including both regions. The white vertical dotted line separates the two regions. High-resolution images of the $\mathbf{b}$ PR and $\mathbf{c}$ EfR. The horizontal white dotted lines in $\mathbf{b}$ and $\mathbf{c}$ represent boundaries between the substrate and film. The reciprocal space images of the $\mathbf{d}$ PR and $\mathbf{e}$ EfR were obtained by fast Fourier transform of the white dashed boxes in $\mathbf{b}, \mathbf{c}$. The red box in $\mathbf{d}$ represents the pseudocubic reciprocal unit cell of LMO.

system with the concentrations of chemical substances changing spatially and temporally due to diffusion, drift and local chemical reactions whereby the substances are transformed into another phase. Similar diffusionreaction models have been employed to describe biological pattern growth ${ }^{49}$, chemical growth ${ }^{50}$ and metal oxidation $^{51}$. More specifically, in our case, we deal with the injected positively charged particles $(\mathrm{P})$ that exhibit catalytic activity transforming the crystalline LMO medium into an amorphous medium. During solid-state amorphization, $\mathrm{P}$ are sometimes stuck in the amorphous medium and can become immobile, thereby decreasing the local density of mobile $\mathrm{P}$ and inducing charged amorphized $\mathrm{LaMnO}_{3}$ substituted by P (a-PLMO), i.e.,

$$
\gamma \mathrm{P}^{+}+\mathrm{LaMnO}_{3} \rightarrow\left[\mathrm{a}-\mathrm{P}_{\gamma} \mathrm{LaMnO}_{3}\right]^{\gamma+},
$$

where $\gamma$ represents the molar ratio of trapped or substituted $\mathrm{P}$ per formula-unit reaction. We regard $\mathrm{P}$ as mobile particles, a-PLMO as a permeable medium, and LMO as an impermeable medium. Since it has been reported that hydration of LMO is thermodynamically unfavorable ${ }^{52}$, only a-PLMO can play the role of a medium for ionic migration of P. Furthermore, the amorphous area in the TEM study was expected to be porous, thus providing broad enough paths for $\mathrm{P}$ to migrate $^{53,54}$.
Therefore, the injected mobile $\mathrm{P}$ diffuse and drift in space-charge-induced electric fields through the a-PLMO medium and can be consumed in the conversion of LMO into a-PLMO (Fig. 4a). With the chemical process, a onedimensional drift-diffusion-reaction model can be set as follows:

$$
\begin{aligned}
& \frac{\partial \mathrm{n}_{\mathrm{P}}}{\partial t}=\frac{\partial}{\partial z}\left(D \frac{\partial \mathrm{n}_{\mathrm{P}}}{\partial z}\right)-\frac{\partial J_{\mathrm{P}}}{\partial z}-k \mathrm{n}_{\mathrm{P}} \mathrm{n}_{\mathrm{LMO}} \\
& \frac{\partial \mathrm{n}_{\mathrm{LMO}}}{\partial t}=-k \mathrm{n}_{\mathrm{P}} \mathrm{n}_{\mathrm{LMO}} \\
& \frac{\partial \mathrm{n}_{\mathrm{a}-\mathrm{PLMO}}}{\partial t}=+k \mathrm{n}_{\mathrm{P}} \mathrm{n}_{\mathrm{LMO}}
\end{aligned}
$$

Here, $n_{\mathrm{P}}, n_{\mathrm{LMO}}$, and $n_{\mathrm{a}-\mathrm{PLMO}}$ represent the number density of the mobile P, LMO, and a-PLMO, and they depend on the elapsed time $t$ and position $z$. $D$ stands for the diffusivity of the $\mathrm{P}$ in the a-PLMO medium. $J_{\mathrm{P}}$ indicates the current density, defined as $J_{\mathrm{P}}=n_{\mathrm{P}} \mu E$, where $\mu$ is the mobility of $\mathrm{P}$ and $E$ is the local electric field at time $t$ and position $z$. The $\mu$ values are coupled with $D$ by the Einstein relation, i.e., $D=\mu k_{\mathrm{B}} T / e$, where $k_{\mathrm{B}}, T$, and $e$ are the Boltzmann constant, temperature, and elementary charge, respectively. $k$ represents the reaction rate constant of the conversion of LMO to a-PLMO by the P. The volume 

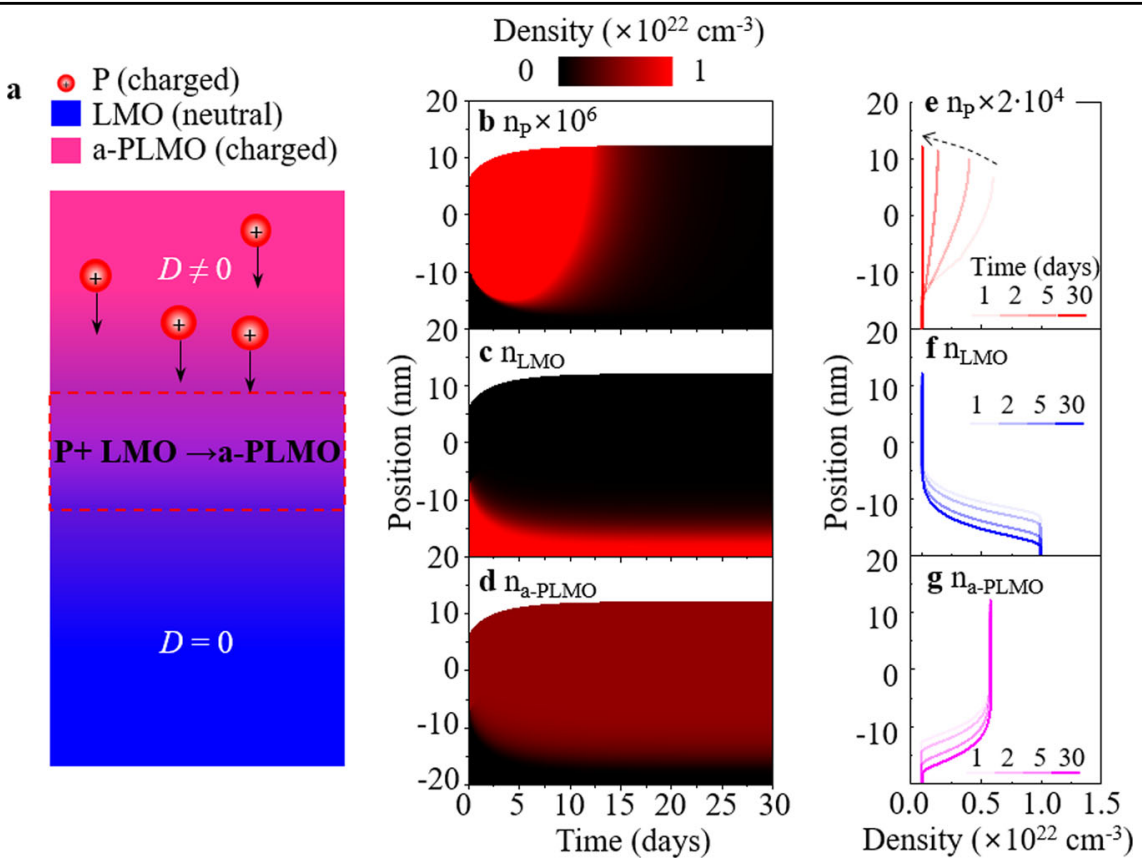

Fig. 4 Representative simulation result of the drift-diffusion-reaction model. a Schematic of the depth profile used for the model. Protons diffuse and drift through the permeable a-PLMO medium and react with the impermeable LMO when they meet at a certain position. Density depth profiles of $\mathbf{b} n_{p}, \mathbf{c} n_{L M O}$, and $\mathbf{d} n_{a-P L M O}$ plotted as a function of elapsed time after the electroforming process. The position ' 0 ' represents the top surface of the film for the PR. $\mathbf{e}-\mathbf{g}$ Density profiles for each element at four selected times. The arrow in e depicts the height increase over time.

expansion was considered by introducing the density ratio ( $\lambda$ ) of LMO to a-PLMO, which was experimentally determined to be $\sim 2$ according to the TEM result. We used the diffusivity of protons experimentally determined in a manganite, i.e., $D \sim 10^{-18} \mathrm{~cm}^{2} \mathrm{~s}^{-155}$.

We performed simulations with settings of $\gamma \sim 10^{-4}$ and $k \sim 10^{-22} \mathrm{~cm}^{3} \mathrm{~s}^{-1}$ (Fig. $4 \mathrm{~b}-\mathrm{g}$ ). This value of $\gamma$ at the given diffusivity of protons can be chosen because it is a primary factor determining the final height at saturation (see Supplementary Fig. 7). On the other hand, the value of $k$ does not make a large difference in the simulations with three orders of magnitude different values, indicating that our simulation is in the diffusion-limited regime, that is, the chemical reaction is fast enough and the slow variation is governed by the diffusion and drift processes of $\mathrm{P}$. In the incipient stage, the relatively high density of $\mathrm{P}$ with the pristine LMO causes a high chemical reaction that converts the impermeable LMO to the permeable aPLMO, thereby leading to a large density gradient of P. This large gradient induces fast diffusion into the interface and consequently stimulates the reaction. The repeated processes result in a rapid increase of the top surface at the initial stage.

The catalytic reactant $\mathrm{P}$ are gradually lost at a rate of one per $\sim 10,000$ chemical reactions at $\gamma \sim 10^{-4}$ and finally disappear, leading to saturation. Figure $4 \mathrm{e}-\mathrm{g}$ shows the rapid reduction in the densities and gradients around the phase boundary. Therefore, the height expansion exponentially decreases with time, and the expansion is saturated after the depletion of $\mathrm{P}$. Although further studies are required to clarify the microscopic origin of the $\mathrm{P}$ permeability in aPLMO, we propose that the biased tip dissociates the water and injects protons similarly to in a previous report ${ }^{21}$. The injected protons undergo diffusion driven by density gradients and drift under space-charge-induced electric fields, and the LMO is amorphized by the chemical reaction with the aid of protons.

\section{Time evolution of the electret}

From the simulation study, we expect that the natural height expansion reaches saturation after approximately 10 days, and the additional increase is as much as $\sim 100 \%$ compared to the initial expansion $(\sim 6 \mathrm{~nm})$ created by the biased tip. In addition, the depth profiles of the positive $\mathrm{P}$ and a-PLMO enable prediction of the time-evolution of the surface potential as well as the value of the potential itself. To confirm our theoretical expectation, we prepared an EfR and carefully observed the topography and surface potential as a function of elapsed time within a month (Fig. 5). Figure 5a, b presents selected topographic images on the 0th and 20th days. The height showed a uniform increase with negligible lateral expansion. The line profiles over time (Fig. 5c) also confirmed the systematic height increase with nearly preserved surface topography. Figure $5 \mathrm{~d}$ summarizes the temporal evolution of the average height, and the experimental results showed 

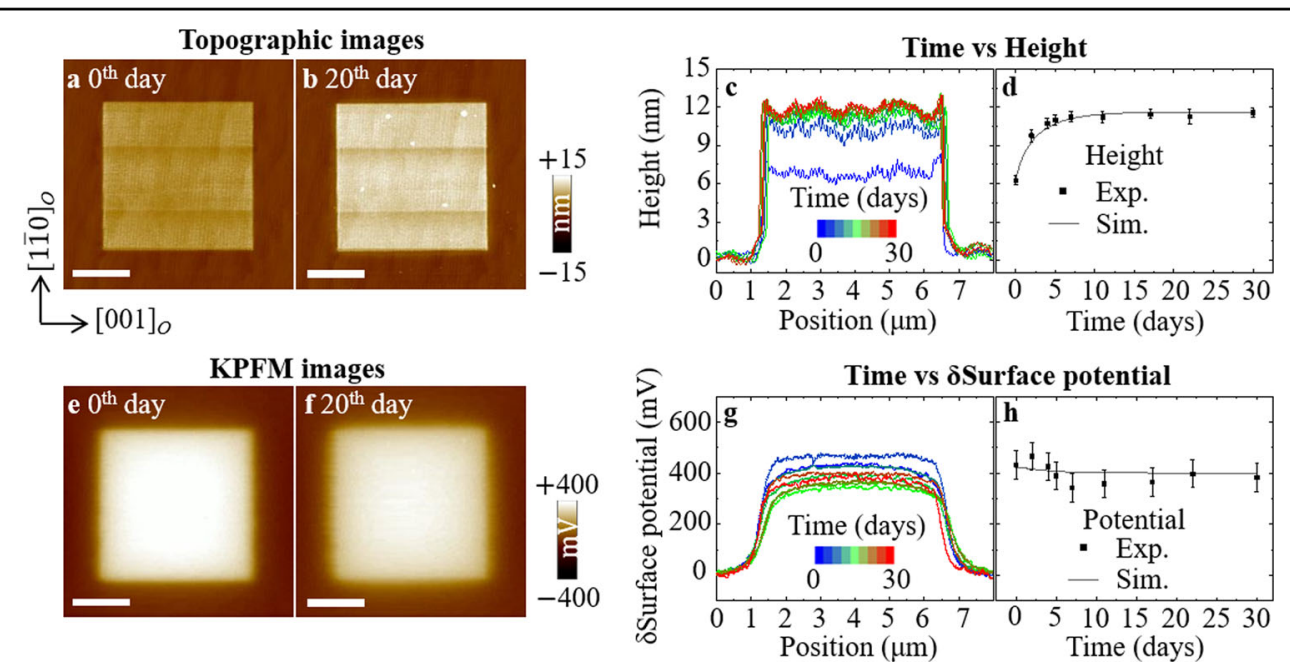

Fig. 5 Temporal evolution of the surface height and surface potential. Topographic images on the $\mathbf{a}$ 0th and $\mathbf{b}$ 20th days after formation. c Height profiles of the EfR, and $\mathbf{d}$ time dependence of the average height. Relative surface potential maps on the $\mathbf{e} 0$ th and $\mathbf{f} 20$ th days. $\mathbf{g}$ Relative surface potential profiles of the EfR, and $\mathbf{h}$ time dependence of the surface potential. The gray lines in $\mathbf{d}, \mathbf{h}$ are the results of the simulation. All scale bars in $\mathbf{a}, \mathbf{b}, \mathbf{e}$, f represent $2 \mu \mathrm{m}$.

excellent agreement with the simulation line. Additionally, we monitored the time dependence of the surface potential for an identical EfR using KPFM (Fig. 5e-h). The surface potential, which is a result of the charge depth profile over the expanded film, exhibited a slight reduction with time of $\sim 400 \mathrm{mV}$, also showing a reasonably good match with the simulation.

\section{Conclusions}

We demonstrated the formation of highly stabilized charge patterns as an electret emerging in a perovskite LMO thin film. By employing SPL, positive charges were injected into the film, thereby inducing solid-state electrochemical amorphization. The charge patterns slowly vertically expanded until the diffusion-limited catalytic reaction and resultant height change were saturated in a week. Additionally, the formed electrostatic charge density was maintained without degradation for more than a year, indicating that the injected charges were still kept therein without significantly escaping from the electret and/or being largely screened by external charges. Based on the drift-diffusion-reaction model in conjunction with the catalytic proton diffusion and trapping hypothesis, we were able to understand the expansion of the amorphous phase and the evolution of charge profiles. Our findings not only introduce a new electret based on TMOs but also provide useful insights into the dynamic features of the electrochemical reaction.

\section{Acknowledgements}

This work was supported by the National Research Foundation (NRF) Grant funded by the Korean Government via the Creative Research Initiative Center for Lattice Defectronics (2017R1A3B1023686).

\section{Author details}

${ }^{1}$ Department of Physics, Korea Advanced Institute of Science and Technology (KAIST), Daejeon 34141, Republic of Korea. ${ }^{2}$ Center for Lattice Defectronics,

KAIST, Daejeon 34141, Republic of Korea. ${ }^{3}$ KAIST Institute for the NanoCentury, KAIST, Daejeon 34141, Republic of Korea

\section{Author contributions}

Y.-J.K. and C.-H.Y. conceived and designed the project. Y.-J.K. performed experiments and simulations. Y.-J.K. and C.-H.Y. wrote the paper.

\section{Conflict of interest}

The authors declare that they have no conflict of interest.

\section{Publisher's note}

Springer Nature remains neutral with regard to jurisdictional claims in published maps and institutional affiliations.

Supplementary information is available for this paper at https://doi.org/ 10.1038/s41427-019-0187-x.

Received: 21 June 2019 Revised: 9 September 2019 Accepted: 17 October 2019.

Published online: 10 January 2020

\section{References}

1. Sessler, G. M. Electrets 2nd edn (Springer, Berlin, 1987).

2. Suzuki, Y. Recent progress in MEMS electret generator for energy harvesting. IEEJ Trans. Electr. Electron. Eng. 6, 101-111 (2011).

3. Kho, T. C., Baker-Finch, S. C. \& McIntosh, K. R. The study of thermal silicon dioxide electrets formed by corona discharge and rapid-thermal annealing. J. Appl. Phys. 109, 53108 (2011).

4. Cuevas, A. et al. Skin care for healthy silicon solar cells. in Proc. 42nd IEEE Photovoltaic Specialist Conference (PVSC) 1-6 (New Orleans, 2015).

5. Bonilla, R. S. \& Wilshaw, P. R. Potassium ions in $\mathrm{SiO}_{2}$ : electrets for silicon surface passivation. J. Phys. D: Appl. Phys. 51, 025101 (2018).

6. Wen, X., Li, D., Tan, K, Deng, Q. \& Shen, S. Flexoelectret: an electret with a tunable flexoelectriclike response. Phys. Rev. Lett. 122, 148001 (2019). 
7. Wu, M.-L., Wang, D. \& Wan, L.-J. Directed block copolymer self-assembly implemented via surface-embedded electrets. Nat. Commun. 7, 10752 (2016).

8. Black, L. E. et al. Explorative studies of novel silicon surface passivation materials: considerations and lessons learned. Sol. Energy Mater. Sol. Cells $\mathbf{1 8 8}$ 182-189 (2018).

9. Cui, J. et al. Titanium oxide: a re-emerging optical and passivating material for silicon solar cells. Sol. Energy Mater. Sol. Cells 158, 115-121 (2016).

10. Mclntosh, K. R. et al. Charge density in atmospheric pressure chemical vapor deposition $\mathrm{TiO}_{2}$ on $\mathrm{SiO}_{2}$-passivated silicon. J. Electrochem. Soc. 156, G190 (2009).

11. Jin, S. et al. Thousandfold change in resistivity in magnetoresistive La-Ca-Mn-O films. Science 264, 413-415 (1994).

12. Dagotto, E. Complexity in strongly correlated electronic systems. Science $\mathbf{3 0 9}$ 257-262 (2005)

13. Suntivich, J., May, K. J., Gasteiger, H. A., Goodenough, J. B. \& Shao-Horn, Y. A perovskite oxide optimized for oxygen evolution catalysis from molecular orbital principles. Science 334, 1383-1385 (2011).

14. Yang, C.-H. et al. Electric modulation of conduction in multiferroic Ca-doped $\mathrm{BiFeO}_{3}$ films. Nat. Mater. 8, 485-493 (2009).

15. Kimura, T. \& Tokura, Y. Layered magnetic manganites. Annu. Rev. Mater. Sci. 30, 451-474 (2000)

16. Lu, N. et al. Electric-field control of tri-state phase transformation with a selective dual-ion switch. Nature 546, 124-128 (2017).

17. Jeen, $\mathrm{H}$. et al. Reversible redox reactions in an epitaxially stabilized $\mathrm{SrCoO}_{x}$ oxygen sponge. Nat. Mater. 12, 1057-1063 (2013).

18. Yao, L., Inkinen, S. \& van Dijken, S. Direct observation of oxygen vacancy-driven structural and resistive phase transitions in $\mathrm{La}_{2 / 3} \mathrm{Sr}_{1 / 3} \mathrm{MnO}_{3}$. Nat. Commun. 8 14544 (2017).

19. Anderson, P. W. Absence of diffusion in certain random lattices. Phys. Rev. 109, 1492-1505 (1957).

20. Xie, X. N., Chung, H. J., Sow, C. H. \& Wee, A. T. S. Nanoscale materials patterning and engineering by atomic force microscopy nanolithography. Mater. Sci. Eng. R. 54, 1-48 (2006)

21. Yang, N. et al. Water-mediated electrochemical nano-writing on thin ceria films. Nanotechnology 25, 075701 (2014).

22. Sirghi, L., Szoszkiewicz, R. \& Riedo, E. Volume of a nanoscale water bridge. Langmuir 22, 1093-1098 (2006).

23. Kim, Y. et al. Local probing of electrochemically induced negative differential resistance in $\mathrm{TiO}_{2}$ memristive materials. Nanotechnology 24, 085702 (2013).

24. Cen, $\mathrm{C}$. et al. Nanoscale control of an interfacial metal-insulator transition at room temperature. Nat. Mater. 7, 298-302 (2008).

25. Strelcov, E. et al. Probing local ionic dynamics in functional oxides at the nanoscale. Nano Lett. 13, 3455-3462 (2013).

26. Li, R.W. AFM lithography and fabrication of multifunctional nanostructures with perovskite oxides. Int. J. Nanotechnol. 6, 1067-1085 (2009).

27. Murakami, $Y$. et al. Resonant $\mathrm{x}$-ray scattering from orbital ordering in $\mathrm{LaMnO}_{3}$. Phys. Rev. Lett. 81, 582-585 (1998).

28. Kim, Y.-J., Lee, J. H., Kim, S. W., Koo, T. Y. \& Yang, C. H. Orientation control of the orbital ordering plane in epitaxqial $\mathrm{LaMnO}_{3}$ thin films by misfit strain. Europhys. Lett. 116, 27003 (2016).

29. Ritter, $C$. et al. Influence of the oxygen content on the structural, magnetotransport, and magnetic properties of $\mathrm{LaMnO}_{3+\delta}$. Phys. Rev. B 56, 8902-8911 (1997).

30. Uehara, M., Mori, S., Chen, C. H. \& Cheong, S.-W. Percolative phase separation underlies colossal magnetoresistance in mixed-valent manganites. Nature 399, 560-563 (1999).

31. Wang, X. R. et al. Imaging and control of ferromagnetism in $\mathrm{LaMnO}_{3} / \mathrm{SrTiO}_{3}$ heterostructures. Science 349, 716-719 (2015).
32. Gilbert, M. et al. Interlayer coupling through a dimensionality-induced magnetic state. Nat. Commun. 7, 11227 (2016).

33. Zhu, Y. et al. Chemical ordering suppresses large-scale electronic phase separation in doped manganites. Nat. Commun. 7, 11260 (2016).

34. Chen, Z. H. et al. Electron accumulation and emergent magnetism in $\mathrm{LaMnO}_{3} /$ $\mathrm{SrTiO}_{3}$ heterostructures. Phys. Rev. Lett. 119, 156801 (2017).

35. May, K. J. et al. Influence of oxygen evolution during water oxidation on the surface of perovskite oxide catalysts. J. Phys. Chem. Lett. 3, 3264-3270 (2012).

36. Risch, M. et al. Structural changes of cobalt-based perovskites upon water oxidation investigated by EXAFS. J. Phys. Chem. C. 117 8628-8635 (2013).

37. Han, B. et al. Activity and stability trends of perovskite oxides for oxygen evolution catalysis at neutral pH. Phys. Chem. Chem. Phys. 17, 22576-22580 (2015).

38. Tan, X. L. et al. Anisotropic-strain-relaxation-induced crosshatch morphology in epitaxial $\mathrm{SrTiO}_{3} / \mathrm{NdGaO}_{3}$ thin films. AlP Adv. 4, 107109 (2014).

39. Chen, H.-T., Raghunath, P. \& Lin, M. C. Computational investigation of $\mathrm{O}_{2}$ reduction and diffusion on $25 \% \mathrm{Sr}$-doped $\mathrm{LaMnO}_{3}$ cathodes in solid oxide fue cells. Langmuir 27, 6787-6793 (2011).

40. Hashiguchi, G., Nakasone, D., Sugiyama, T., Ataka, M. \& Toshiyoshi, H. Charging mechanism of electret film made of potassium-ion-doped $\mathrm{SiO}_{2}$. AlP Adv. $\mathbf{6}$ 035004 (2006).

41. Sugiyama, $\mathrm{T}$. et al. $\mathrm{SiO}_{2}$ electret generated by potassium ions on a comb-drive actuator. Appl. Phys. Express 4, 114103 (2011).

42. Bonilla, R. S. \& Wilshaw, P. R. A technique for field effect surface passivation for silicon solar cells. Appl. Phys. Lett. 104, 232903 (2014).

43. Tong, S. et al. Mechanical removal and rescreening of local screening charges at ferroelectric surfaces. Phys. Rev. Appl. 3, 014003 (2015).

44. Sharma, P. et al. Oxygen stoichiometry effect on polar properties of $\mathrm{LaAlO}_{3} /$ $\mathrm{SrTiO}_{3}$. Adv. Funct. Mater. 28, 1707159 (2018).

45. Mélin, T., Diesinger, H., Deresmes, D. \& Stiévenard, D. Electric force microscopy of individually charged nanoparticles on conductors: an analytical model for quantitative charge imaging. Phys. Rev. B. 69, 035321 (2004).

46. Lee, K. Y. et al. Controllable charge transfer by ferroelectric polarization mediated triboelectricity. Adv. Funct. Mater. 26, 3067-3073 (2016).

47. Mondal, P., Bhattacharya, D., Choudhury, P. \& Mandal, P. Dielectric anomaly at $T_{N}$ in $\mathrm{LaMnO}_{3}$ as a signature of coupling between spin and orbital degrees of freedom. Phys. Rev. B. 76, 172403 (2007).

48. Young, M. J., Holder, A. M., George, S. M. \& Musgrave, C. B. Charge storage in cation incorporated a- $\mathrm{MnO}_{2}$. Chem. Mater. 27, 1172-1180 (2015).

49. Kondo, S. \& Miura, T. Reaction-diffusion model as a framework for understanding biological pattern formation. Science 329, 1616-1620 (2010).

50. Mikhailov, A. S. \& Showalter, K. Control of waves, patterns and turbulence in chemical systems. Phys. Rep. 425, 79-194 (2006).

51. Hauffe, K. Oxidation of metals. (Plenum Press, New York, 1965).

52. Norby, T. Proton conduction in solids: bulk and interfaces. MRS Bull. 34, 923-928 (2009).

53. $\mathrm{Ma}, \mathrm{H}$. et al. Cationic covalent organic frameworks: a simple platform of anionic exchange for porosity tuning and proton conduction. J. Am. Chem. Soc. 138, 5897-5903 (2016).

54. Gregori, G., Shirpour, M. \& Maier, J. Proton conduction in dense and porous nanocrystalline ceria thin films. Adv. Funct. Mater. 23, 5861-5867 (2013).

55. Ouksel, L., Kerkour, R. \& Chelali, N. E. Proton diffusion process manganese dioxide for use in rechargeable alkaline zinc manganese dioxide batteries and its electrochemical performance. lonics 22, 1751 (2016). 